\title{
RDF Analysis of Small-Signal Equivalent Circuit Parameters in MOSFET Devices
}

\author{
L. Oniciuc and P. Andrei \\ Department of Electrical and Computer Engineering, Florida State University, \\ Tallahassee, FL, USA \\ \{oniciuc|pandrei\}@eng.fsu.edu
}

\begin{abstract}
A perturbation technique is developed for the analysis of random doping induced fluctuations (RDF) of small-signal equivalent circuit parameters in semiconductor devices. This technique is based on the computation of the doping sensitivity functions of parameters of interest by using the admittance matrix parameters and is applied to the study of RDF of equivalent circuit parameters in a 40-nm channel length MOSFET. The presented technique can be easily extended to the analysis of RDF in other semiconductor devices such as SOI, HEMT, etc.
\end{abstract}

\section{Introduction}

There are many studies in the literature related to the analysis of random doping induced fluctuation (RDF) effects in nanoscale semiconductor devices. Most of the existing studies focus on the investigation of fluctuations of terminal currents $[1,2]$, threshold voltages [2, 3] and capacitances in MOSC structures, subthreshold characteristics [4], and static-noise margins [5]. Although there is some work related to the analysis of fluctuations of small-signal parameters in semiconductor devices, the existing work focuses exclusively on the analysis of y-parameters [6], and there is no effort related to the analysis of fluctuations of small-signal equivalent circuit parameters (SSCP) such as the ones represented in Fig. 1a for a MOSFET device. However, the accurate investigation of the fluctuations of these parameters is extremely important for circuit design purposes, particularly of high-frequency analog circuits, such as LNAs or operational amplifiers. In this article we present for the first time an analysis of the RDF of SSCP in nanoscale MOSFET devices based on a perturbation technique. Section 2 describes the basics of our technique, while sample numerical results and conclusions are drawn in Section 3.

\section{Technical discussion}

Following the linearization method presented in $[2,6]$, the fluctuations of any circuit parameter $A$ (such as $g_{m}, C_{g s}, C_{g d}$, etc.) can be written in the first-order approximation as a linear combination of doping variations $\tilde{D}(\boldsymbol{r})$ :

$\tilde{A}=\int_{\Omega} \gamma_{A}(\boldsymbol{r}) \tilde{D}(\boldsymbol{r}) d V$ 
where $\tilde{D}(\boldsymbol{r})$ is the infinitesimal variation of the doping concentration at location $\boldsymbol{r}$, and $\gamma_{A}(\boldsymbol{r})$ is the doping sensitivity function of parameter $A$. The integral in eq. (1) is taken over the whole volume of the semiconductor device $\Omega$. If one knows the doping sensitivity function of some parameter, one can compute the variance of that parameter by using $\sigma_{A}^{2}=\int_{\Omega} \gamma_{A}^{2}(\boldsymbol{r}) D(\boldsymbol{r}) d V$, where $D(\boldsymbol{r})$ is the average doping concentration at location $\boldsymbol{r}$. The computation of the doping sensitivity functions of SSCP is in general a difficult task, which involves the simulation of many devices with various doping concentration distributions. Below, we present a much simpler solution to the computation of the sensitivity functions of SSCP based on the variations of admittance matrix parameters (y-parameters): $y_{i j}=\partial I_{i} /\left.\partial V_{j}\right|_{V_{k}=\text { const. }}$, $k \neq j$, where $i, j$, and $k$ denote the electrodes of the device.

Suppose that we have already computed the values of $y_{i j}$ as well as of their doping sensitivity functions, $\gamma_{y_{i j}}(\boldsymbol{r})$. For a detailed analysis on how to compute the sensitivity functions of y-parameters we refer to [6]. Now note that the SSCP of most semiconductor devices can be written as a function of $y_{i j}$. For instance, in the case of MOSFETs described by the small-signal equivalent circuit shown in Fig. 1a:

$$
\begin{aligned}
& C_{d s}=-\frac{\operatorname{Im}\left(y_{d d}+y_{g d}\right)}{\omega}, \quad C_{g s}=-\frac{\operatorname{Im} y_{g g}+\operatorname{Im} y_{g d}}{\omega}\left[1+\left(\frac{\operatorname{Re} y_{g g}}{\operatorname{Im} y_{g g}+\operatorname{Im} y_{g d}}\right)^{2}\right], \\
& C_{g d}=-\frac{\operatorname{Im} y_{g s}}{\omega}, \quad R_{i}=\frac{\operatorname{Re} y_{g g}}{\left(\operatorname{Im} y_{g g}-\omega C_{g d}\right)^{2}+\left(\operatorname{Re} y_{g g}\right)^{2}}, g_{d s}=\operatorname{Re} y_{d d}, \text { etc. },
\end{aligned}
$$

where $\omega$ is the angular frequency, Im and Re denote the imaginary and real parts of the given quantities. Next let us compute the doping sensitivity functions of the parameters in the left-hand sides of equations (1a-e) as a function of $\gamma_{y_{i j}}(\boldsymbol{r})$. Any fluctuation $\tilde{D}(\boldsymbol{r})$ of the doping concentration leads to variations $\tilde{y}_{i j}$ of y-parameters. Variations of y-parameters result in variations of SSCP that can be computed by using equations (2). For instance, linearizing (2) with respect to the variations of yparameters, one can show that: $\tilde{C}_{d s}=-\frac{\operatorname{Im}\left(\tilde{y}_{d d}+\tilde{y}_{g d}\right)}{\omega}, \quad \tilde{C}_{g d}=-\frac{1}{\omega} \operatorname{Im} \tilde{y}_{g s}$, $\tilde{C}_{g s}=-\frac{\operatorname{Im}\left(\tilde{y}_{g g}+\tilde{y}_{g d}\right)}{\omega}\left(1+\Delta^{2}\right)+\frac{2 \Delta}{\omega}\left[\operatorname{Re} \tilde{y}_{g g}-\operatorname{Re} y_{g g} \frac{\operatorname{Im}\left(\tilde{y}_{g g}+\tilde{y}_{g d}\right)}{\operatorname{Im}\left(y_{g g}+y_{g d}\right)}\right], \quad$ etc., where $\Delta=\frac{\operatorname{Re} y_{g g}}{\operatorname{Im}\left(y_{g g}+y_{g d}\right)}$. The doping sensitivity functions of SSCP can be computed by introducing (1) in the last equations and identifying the sensitivity functions: 

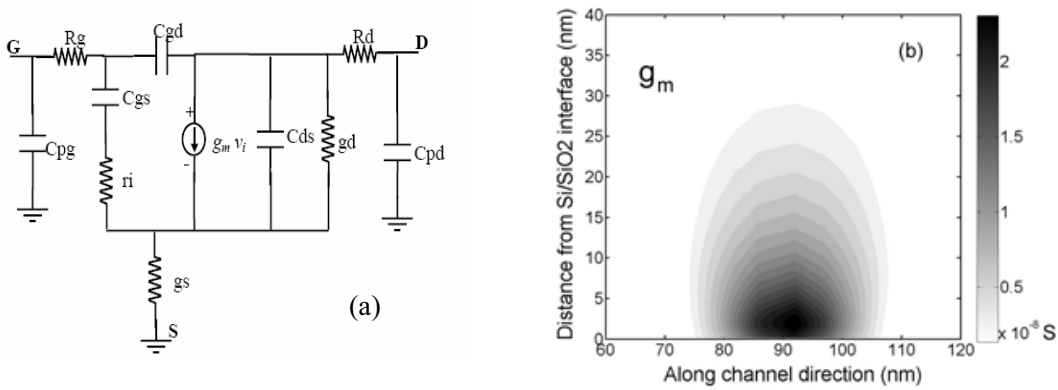

Figure 1: (a) Small-signal equivalent circuit; small-signal parameters $C_{g s}, R_{g}, g_{m}$, etc. fluctuate due to RDF. (b) Doping sensitivity function of $g_{m}$.
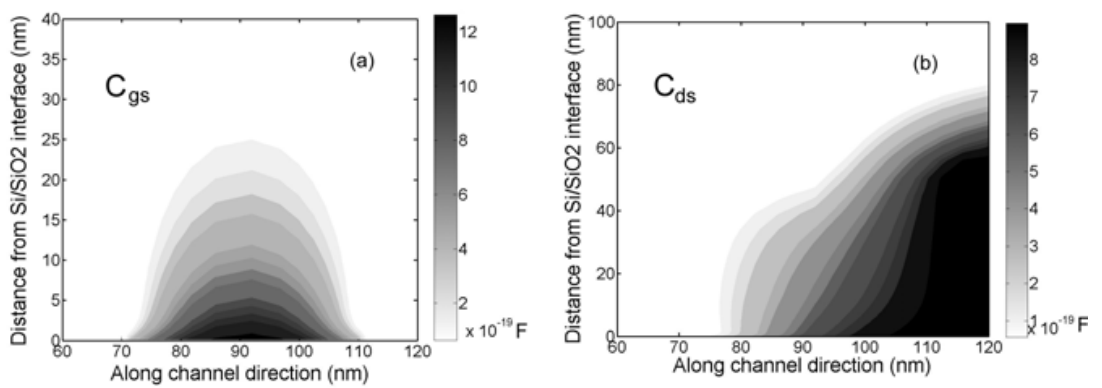

Figure 2: Doping sensitivity function of $C_{g s}$ (a) and $C_{d s}$. (b) The channel extends from 70 to $110 \mathrm{~nm}$ in the along channel direction. The dark regions are regions inside the MOSFET device that are particularly sensitive to RDF.
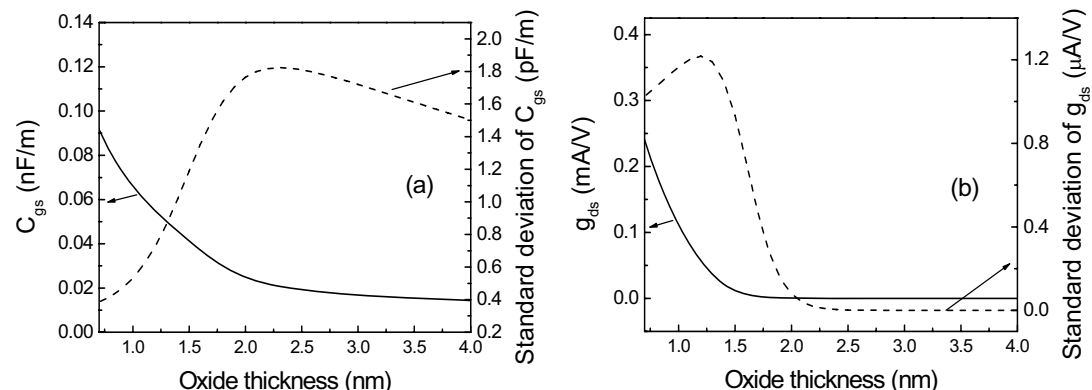

Figure 3: (a) $C_{g s}$ (left axis) and $\sigma_{C_{g s}}$ (right axis) as a function of the oxide thickness. (b) Drain-to-source channel conductance (left axis) and standard deviation of $g_{d s}$ (right axis) as a function of the oxide thickness. 


$$
\begin{aligned}
& \gamma_{C_{d s}}=-\frac{\operatorname{Im}\left(\gamma_{y_{g s}}+\gamma_{y_{g s}}\right)}{\omega}, \quad \gamma_{C_{g d}}=-\frac{1}{\omega} \operatorname{Re} \gamma_{y_{g d}}, \gamma_{g_{d s}}=\operatorname{Re} \gamma_{y_{d d}} \text {, and } \\
& \gamma_{C_{g s}}=-\frac{\operatorname{Im}\left(\gamma_{y_{g g}}+\gamma_{y_{g d}}\right)}{\omega}\left(1+\Delta^{2}\right)+\frac{2 \Delta}{\omega}\left[\operatorname{Re} \gamma_{y_{g g}}-\operatorname{Re} \gamma_{y_{g g}} \frac{\operatorname{Im}\left(\gamma_{y_{g g}}+\gamma_{y_{g d}}\right)}{\operatorname{Im}\left(y_{g g}+y_{g d}\right)}\right] .
\end{aligned}
$$

Similar equations can be written for the sensitivity functions of the other SSCP.

\section{Numerical results}

The technique presented in the previous section has been numerically implemented in our device simulator RandFlux [7]. For illustration purposes, we present a few sample results obtained for a $40 \mathrm{~nm}$ channel length MOSFET with simplified architecture. The doping concentrations of the drain, source, and polygate are $5 \times 10^{19} \mathrm{~cm}^{-3}$, the oxide thickness is $1.8 \mathrm{~nm}$, the doping concentration in the substrate is constant and equal to $5 \times 10^{18} \mathrm{~cm}^{-3}$, and the diffusion of the drain and source junctions under the gate are $2 \mathrm{~nm}$. Quantum mechanical effects were taken into account in this analysis by the Density-Gradient model [8]. The width of the device is $W=1 \mu \mathrm{m}$, but the results presented in this section can be easily scaled down to lower dimensions. The doping sensitivity functions are represented in Fig. $1 \mathrm{~b}, 2 \mathrm{a}$ and $2 \mathrm{~b}$ for $g_{m}, C_{g s}$, and $C_{d s}$, respectively. This sensitivity functions show how sensitive the SSCP are to RDF and, hence, they can be used in the design of semiconductor devices with reduced random doping induced fluctuations (fluctuations resistant structures). It is remarkable that the locations of the regions that are highly sensitive to RDF depend on the nature of the parameter. For most parameters these regions are located more or less close to the middle point of the conduction channel, but for some parameters (such as $C_{d s}$ ) the most sensitive region is located towards the drain region.

In Figs. 3a-b the standard deviations of $C_{g s}$ and $g_{m}$ are represented as a function of the oxide thickness for $V_{d s}=0.1 \mathrm{~V}, f=1 \mathrm{GHz}$, and $V_{g s}$ is $0.2 \mathrm{~V}$ above the threshold voltage. The fluctuations of $C_{g s}$ and most other small-signal equivalent circuit parameters are larger when the gate voltage is approximately equal to the threshold voltage (in the simulations presented in Fig. 3 this happens at $t_{o x} \approx 1.8 \mathrm{~nm}$ ).

\section{References}

[1] A. Wettstein, O. Penzin, E. Lyumkis, and W. Fichner, SISPAD 2004 Proc., pp. 91.

[2] I.D. Mayergoyz and P. Andrei, J. Appl. Phys., vol. 90, pp. 3019, 2001.

[3] A. Asenov, A. R. Brown, J.H. Davies, S. Kaya, and S. Slavcheva, IEEE Trans. Electron Devices, vol. 50, pp. 1837, 2003.

[4] P. Andrei and I.D. Mayergoyz, Solid-State Electron., vol. 47, pp. 2055, 2003

[5] B. Cheng, S. Roy, A. Asenov, Int. Conf.-MIXDES, June 2006.

[6] P. Andrei and I.D. Mayergoyz, J. Appl. Phys., vol. 96, pp. 2071, 2004.

[7] RandFlux, http://www.eng.fsu.edu/ pandrei/RandFlux/.

[8] M. G. Ancona and G. J. Iafrate, Phys. Rev. B, vol. 39, pp. 9536, 1989. 